\title{
The ASTRODEEP Frontier Fields catalogues
}

\section{Multiwavelength photometry and rest-frame properties of MACS-J0717 and MACS-J1149}

\author{
M. Di Criscienzo ${ }^{1}$, E. Merlin ${ }^{1}$, M. Castellano ${ }^{1}$, P. Santini ${ }^{1}$, A. Fontana ${ }^{1}$, R. Amorin ${ }^{2,3}$, K. Boutsia ${ }^{4}$, S. Derriere ${ }^{7}$, \\ J. S. Dunlop ${ }^{5}$, D. Elbaz ${ }^{6}$, A. Grazian ${ }^{1}$, R. J. McLure ${ }^{5}$, E. Mármol-Queraltó ${ }^{5}$, M. J. Michalowski ${ }^{5}$, S. Mortlock ${ }^{5}$, \\ S. Parsa ${ }^{5}$, and L. Pentericci ${ }^{1}$ \\ 1 INAF-Osservatorio Astronomico di Roma, via Frascati 33, 00040 Monte Porzio Catone (RM), Italy \\ e-mail: dicrisci@gmail.com \\ 2 Cavendish Laboratory, University of Cambridge, 19 JJ Thomson Avenue, Cambridge, CB3 OHE, UK \\ ${ }^{3}$ Kavli Institute for Cosmology, University of Cambridge, Madingley Road, Cambridge CB3 OHA, UK \\ 4 Carnegie Observatories, Colina El Pino, Casilla 601 La Serena, Chile \\ 5 SUPA, Institute for Astronomy, University of Edinburgh, Royal Observatory, Edinburgh, EH9 3HJ, UK \\ ${ }^{6}$ Laboratoire AIM-Paris-Saclay, CEA/DSM/Irfu-CNRS-Université Paris-Saclay, pt courrier 131, 91191 Gif-sur-Yvette, France \\ 7 Observatoire Astronomique de Strasbourg, Université de Strasbourg, CNRS, UMR 7550, 11 rue de l'Université, \\ 67000 Strasbourg, France
}

Received 15 May 2017 / Accepted 22 September 2017

\begin{abstract}
Aims. We present the multiwavelength photometry of two Frontier Fields massive galaxy clusters, MACS-J0717 and MACS-J1149, and their parallel fields, ranging from Hubble Space Telescope (HST) to ground-based $K$ and Spitzer IRAC bands, and the public release of photometric redshifts and rest-frame properties of galaxies found in cluster and parallel pointings. This work was carried out within the ASTRODEEP project and aims to provide a reference for future investigations of extragalactic populations.

Methods. To fully exploit the depth of the images and detect faint sources, we used an accurate procedure, which carefully removes the foreground light of bright cluster sources and the intra-cluster light thus enabling detection and measurement of accurate fluxes in crowded cluster regions. This same procedure has been successfully used to derive the photometric catalogue of MACS-J0416 and Abell-2744.

Results. The obtained multiband photometry was used to derive photometric redshifts, magnification, and physical properties of sources. In line with the first two FF catalogues released by ASTRODEEP, the photometric redshifts reach $\sim 4 \%$ accuracy. Moreover we extend the presently available samples to galaxies intrinsically as faint as $H 160 \sim 32-34$ mag thanks to the magnification factors induced by strong gravitational lensing. Our analysis allows us to probe galaxy masses larger than $10^{7} M_{\odot}$ and $/$ or $S F R=0.1-1 M_{\odot} / \mathrm{yr}$ out to redshift $z>6$.
\end{abstract}

Key words. galaxies: distances and redshifts - galaxies: high-redshift - catalogs

\section{Introduction}

The Hubble Frontier Fields (FF) programme (Lotz et al. 2017) has been conceived and designed to explore the highest redshift Universe down to the faintest rest-frame luminosities attainable before the launch of the James Webb Space Telescope (JWST); this FF programme combines the capabilities of the Hubble Space Telescope (HST) with the amplification power of massive galaxy clusters. The programme (PI. Lotz), which was started in 2012 using HST director discretionary time, has devoted 560 orbits $(\sim 630 \mathrm{~h})$ to observe six clusters of galaxies. The FF target clusters were selected as six of the most powerful gravitational lenses presently known, providing lensing amplifications of typically 2 over a significant fraction of the WFC3 field of view up to 10-50 in the most extreme cases.

The HST images are supplemented by a wealth of data including Spitzer and ground-based imaging and spectroscopic follow-up. The key science driver of the FF programme is shedding light on the properties of galaxies at high redshift $(z>5)$, which are critically important for our understanding of the processes involved in the reionization of the Universe and are presently constrained only from the brightest galaxies discovered in blank-field surveys (Castellano et al. 2016b; Menci et al. 2016; Bouwens et al. 2017, 2016; McLeod et al. 2016; Vanzella et al. 2017a,b; Livermore et al. 2017; Wei et al. 2017).

To achieve the ambitious goal of probing the distant universe to an unprecedented depth it is important to develop accurate photometric procedures that reveal the power of the deepest images. This is the main scope of the European FP7-Space project ASTRODEEP, a coordinated and comprehensive programme of i) algorithm and software development and testing; ii) data reduction and release, and iii) scientific data validation and analysis of the deepest multiwavelength cosmic surveys ${ }^{1}$.

In the first two papers, Merlin etal. (2016a) and Castellano et al. (2016a), we described the procedures developed within this collaboration to produce multiband and

1 For more information visit http://astrodeep.eu 
Table 1. PSF FWHM and depths of the dataset (see text).

\begin{tabular}{|c|c|c|c|c|}
\hline \multirow[t]{2}{*}{ Image } & PSF $F W H M\left({ }^{\prime \prime}\right)$ & Limiting $\mathrm{AB}$ magnitude & PSF $F W H M\left({ }^{\prime \prime}\right)$ & Limiting AB magnitude \\
\hline & \multicolumn{2}{|c|}{ M0717 Cluster } & \multicolumn{2}{|c|}{ M0717 Parallel } \\
\hline ACS B435 & 0.11 & 28.64 & 0.10 & 28.71 \\
\hline ACS V606 & 0.13 & 28.67 & 0.12 & 28.92 \\
\hline ACS $I 814$ & 0.16 & 28.99 & 0.14 & 29.13 \\
\hline WFC3 $Y 105$ & 0.16 & 29.33 & 0.17 & 28.94 \\
\hline WFC3 J125 & 0.18 & 28.98 & 0.18 & 28.96 \\
\hline WFC3 JH140 & 0.18 & 29.02 & 0.18 & 28.97 \\
\hline WFC3 $H 160$ & 0.18 & 29.06 & 0.17 & 28.97 \\
\hline MOSFIRE $K s$ & 0.4 & 25.08 & 0.4 & 25.19 \\
\hline IRAC 3.6 & 1.66 & 25.47 & 1.66 & 25.22 \\
\hline \multirow[t]{2}{*}{ IRAC 4.5} & 1.72 & 25.22 & 1.72 & 25.19 \\
\hline & \multicolumn{2}{|c|}{ M1149 Cluster } & \multicolumn{2}{|c|}{ M1149 Parallel } \\
\hline ACS B435 & 0.11 & 28.30 & 0.10 & 28.26 \\
\hline ACS V606 & 0.12 & 28.88 & 0.10 & 28.71 \\
\hline ACS $I 814$ & 0.15 & 29.08 & 0.13 & 28.90 \\
\hline WFC3 $Y 105$ & 0.15 & 29.25 & 0.17 & 29.33 \\
\hline WFC3 J125 & 0.17 & 29.12 & 0.16 & 29.02 \\
\hline WFC3 JH140 & 0.19 & 28.72 & 0.17 & 29.02 \\
\hline WFC3 $H 160$ & 0.17 & 29.18 & 0.17 & 29.09 \\
\hline MOSFIRE $K s$ & 0.5 & 24.65 & 0.5 & 24.52 \\
\hline IRAC 3.6 & 1.66 & 25.41 & 1.66 & 25.08 \\
\hline IRAC 4.5 & 1.72 & 25.71 & 1.72 & 25.21 \\
\hline
\end{tabular}

photometric redshift catalogues and their application to the first two released FF Abell-2744 and MACS-J0416.

In this paper, we present the public release of the multiwavelength photometry of MACS-J0717+3745 and MACSJ1149.5+2223 (hereafter M0717 and M1149), which include both HST ACS and WFC3, Keck-MOSFIRE Ks-band, and Spitzer-IRAC observations.

The paper is structured as follows: in Sect. 2 we describe the dataset used in this study; Sect. 3 gives a short description of the procedure we applied to obtain the detection catalogue and photometric measurements in optical and NIR bands. In Sect. 4 we present the released catalogue describing in particular the procedure used to compute the photometric redshifts, magnification, and rest-frame galaxy properties. Conclusions close the paper.

In the following we adopt the $\Lambda$-CDM concordance cosmological model $\left(H_{0}=70 \mathrm{~km} \mathrm{~s}^{-1} \mathrm{Mpc}^{-1}, \Omega_{\mathrm{M}}=0.3\right.$ and $\left.\Omega_{\lambda}=0.7\right)$. All magnitudes are in $\mathrm{AB}$ system unless explicitly mentioned.

\section{The dataset}

M0717 and M1149 are the third and fourth of a total of six twin fields observed by HST in seven optical and near-infrared bands: $F 435 W, F 606 W$, and $F 814 W$ from ACS/WFC and $F 105 W$, $F 125 W, F 140 W$, and $F 160 W$ from WFC3/IR. Each of these fields is observed by HST in parallel mode, i.e. cluster and a blank adjacent field.

We used the final reduced and calibrated v1.0 mosaics released by STScI, drizzled at $0.06^{\prime \prime}$ pixel-scale. A detailed description of the acquisition strategy and of the data reduction pipeline can be found in the STScI data release documentation $^{2}$. We also include the Keck/MOSFIRE $K s$ images from Brammer et al. (2016) and the IRAC 3.6 and 4.5 data acquired by Spitzer under Director Discretionary time (PI Capak).

In Table 1 we list PSF FWHM and limiting magnitudes of the dataset. For the HST images the depths have been computed

\footnotetext{
2 https://archive.stsci.edu/pub/hlsp/frontier/
}

as the magnitudes within a circular aperture of two times the FWHM of $5 \sigma$ detections in the $H 160$ images, as measured by SExtractor on PSF-matched images. To estimate the depths of the MOSFIRE and IRAC images, we use the corrected rms maps (see below) computing $f_{5 \sigma}=5 \cdot \sqrt{A_{\text {aper }}} \cdot f_{\text {rms }}$ in each pixel, where $A_{\text {aper }}$ is the area of a circular region with radius equal to the PSF FWHM, and taking the mode of the distributions as the final value.

\section{Multiwavelength photometry}

\subsection{Removing the ICL and bright cluster members}

To fully exploit the depth of the images and detect faint sources we used an accurate procedure to remove the foreground light of bright cluster sources and the intra-cluster light (ICL). This procedure is described in detail in Merlin et al. (2016a); it is even more necessary, compared to the previously studied FFs for M0717 and M1149, in which few multiple merging and subclusters were present making the ICL bright and patchy ${ }^{3}$. In brief, we initially estimated a first-guess model for the ICL component masking $S / N>10$ pixels and we fitted the diffuse light with a Ferrer (Binney \& Tremaine 1987) profile centred on the mass centre of the whole cluster. Then on the ICL-subtracted $H 160$ image, with an iterative method that uses both Galapagos (Barden et al. 2012) and Galfit ${ }^{4}$ (Peng et al. 2011), we derived a one- and two-component fit of the brightest cluster galaxies. Finally, we used these fits to refine the model of ICL and produced the residual image (see Fig. 1), where the patchy ICL and the light from bright sources are subtracted.

Unlike in MACS-J0416 and Abell-2744, where all bright galaxies were Galfit-ed with two components to fit the central

3 This step is obviously unnecessary in the case of parallel fields.

4 Galapagos and Galfit are two public data analysis algorithms that fit 2D analytic functions to galaxies and point sources directly to digital images. 


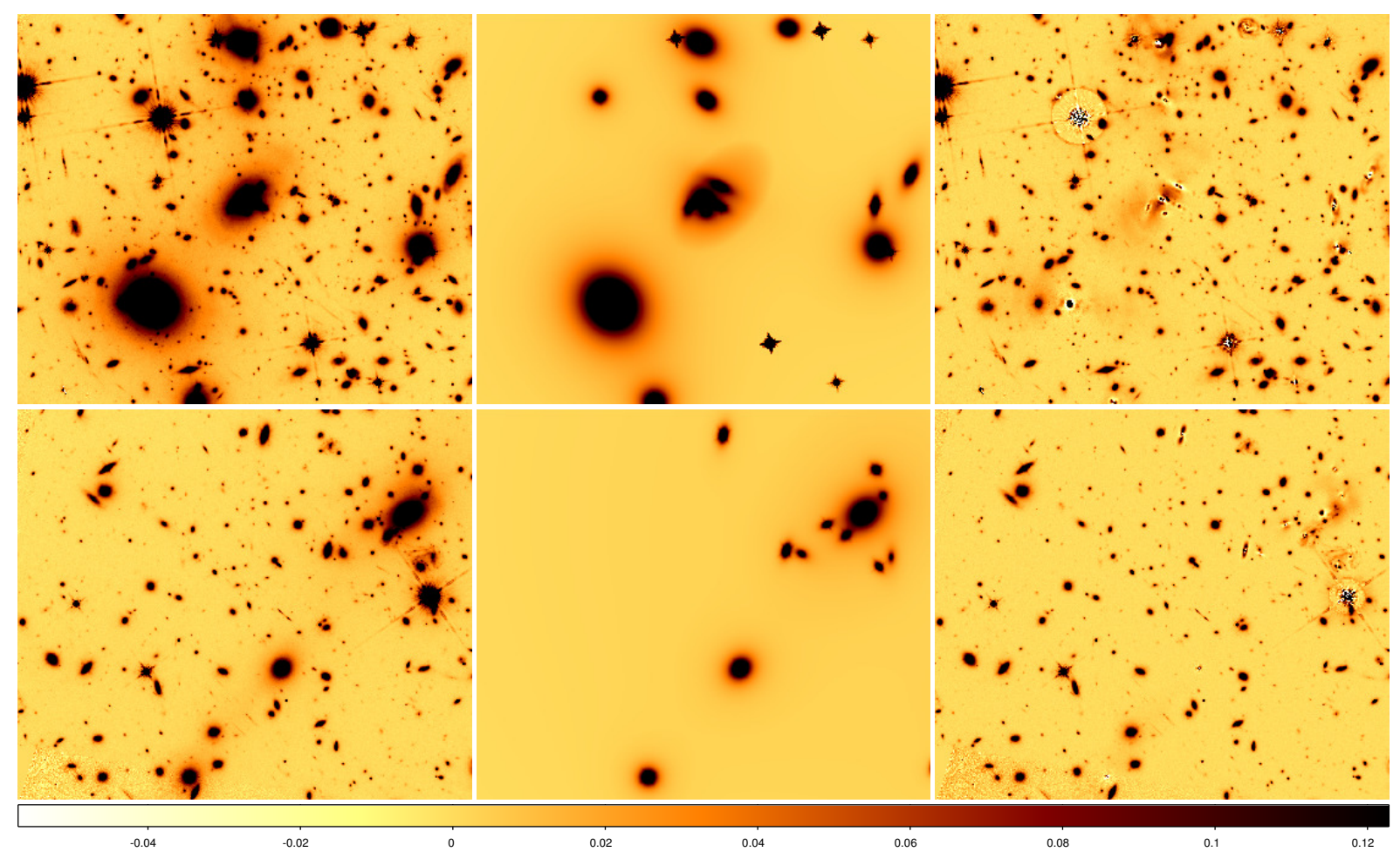

Fig. 1. Basic steps performed on the H160 images to remove the light of the cluster sources of M0717 (upper panels) and M1149 (lower panels). From the left to the right: original images, final and refined Galfit models of bright objects and ICL, and final residual image (=observed - model) after median filtering are shown. All the images are in linear scale scale with the same cuts.

regions accurately (Merlin et al. 2016a), here we found a better solution in the form of a flatter residual image using a single component, or very faint second one, for those bright galaxies located in the crowded regions (subclusters). Instead, during the ICL refinement fit, we added a second component to the ICL Ferrer profile centred on the subclusters.

In both clusters there is a saturated star in the central part of the $H$ image whose light must be removed to produce accurate photometry of the faint galaxies. To do this, we subtracted most of the light from the saturated star with an ad hoc PSF model. To build this model, we constructed the median image constructed from the star itself with three of its rotations of 90, 180, and 270 degrees in order to remove the brilliant objects nearby the stars. Finally a median filtering was applied to remove the remaining intermediate scale background residuals. As demonstrated in Merlin et al. (2016a) the detection on these residual image, as opposed to the detection on the original images, enables a more efficient recovery of the faint sources.

We applied the same procedure to all the other HST bands. For consistency and to reduce the computing times, we sequentially moved from the $H$ band to the bluer bands. We adopted, as a first-guess parameter for both ICL and bright cluster galaxies, the best-fit parameter of the band immediately redward of that band; for example, we used the $H 160$ band parameters as first guess to fit the ICL and bright sources in the JH140 band, those of $J H 140$ when fitting the $J 125$, and so on.

As a final refining step, to take into account the effect of the subtracted sources on the detection and the flux measurement in the innermost cluster regions, we inserted photon noise of the Galfit-subtracted images in the original rms map. This step was performed summing in quadrature the variance of the original rms map with that of a photon noise image obtained from Galfit model image and image exposure times.

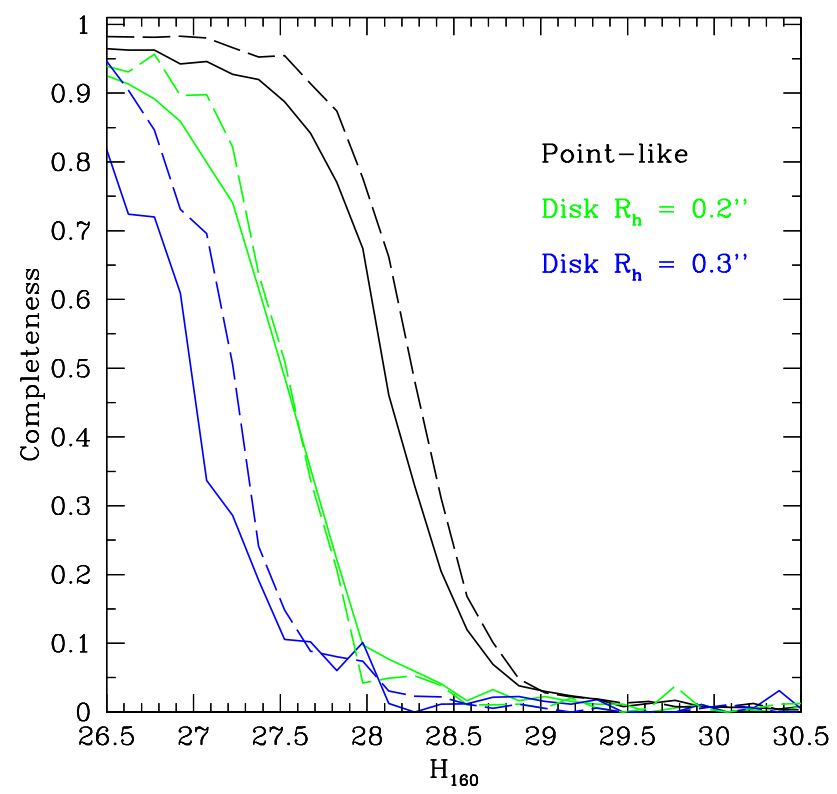

Fig. 2. Completeness of the $H$-detected catalogue for point-like and disk-like sources in M0717 (solid lines) and M1149 (dashed lines).

\subsection{Detection catalogue and HST photometry}

The detection catalogue was produced in two steps. First, we used SExtractor (Bertin et al. 1996) on the processed H160 image with a revised HOT+COLD approach (Galametz et al. 2013; Guo et al. 2013). We then added the additional objects detected in a median average of the $Y 105+J 125+J H 140+H 160$ bands, which are undetected in the $H$ band. This last step more effectively identifies very blue galaxies close to the detection limit of the images, which are expected to include a good fraction of 

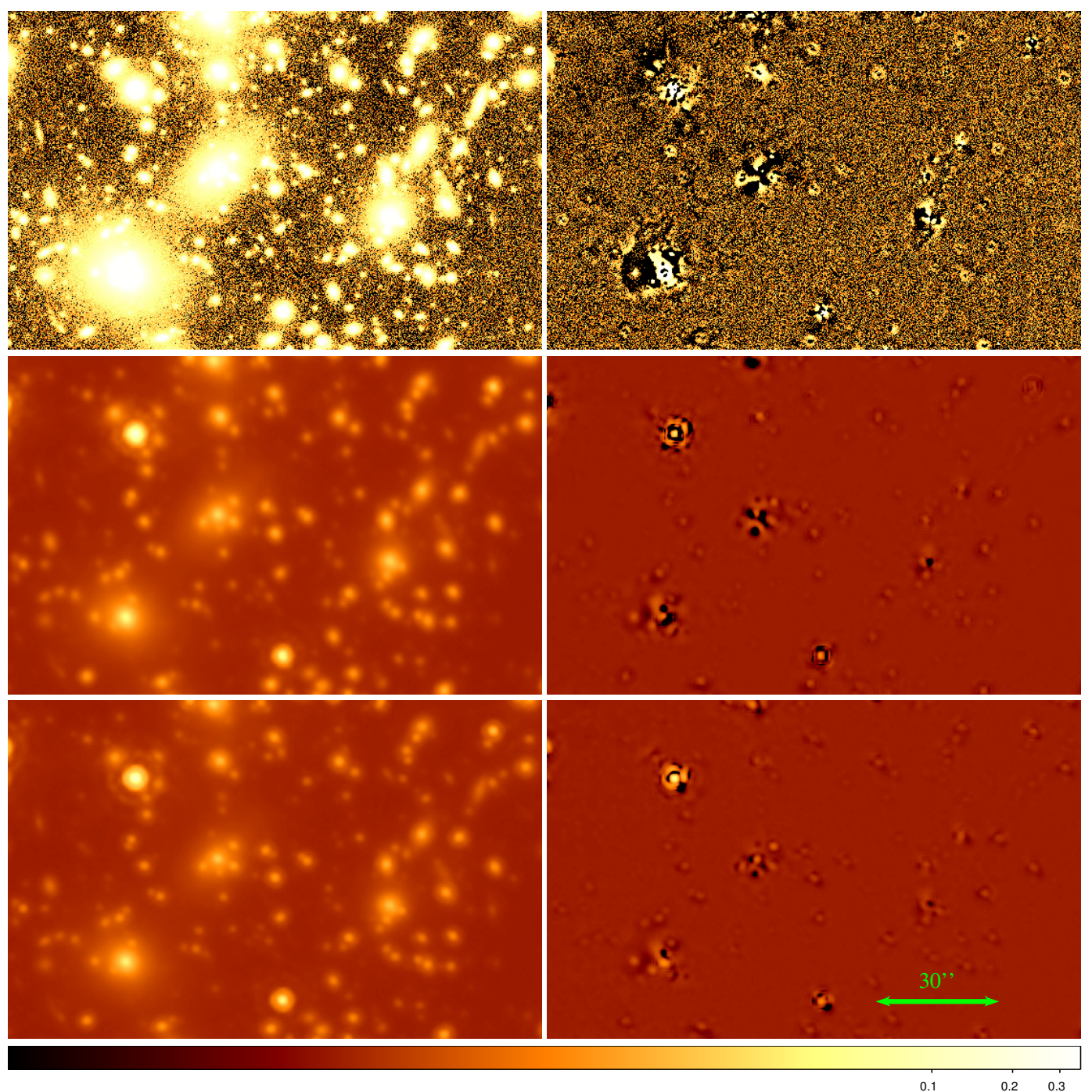

Fig. 3. Original (left) and residual (right) images in $K$ (upper panels), IRAC-CH1 (central panels), and IRAC-CH2 (lower panels) bands of M0717 after processing with T-PHOT (see Sect. 3.3 for details). All images (original and residuals) have logarithmic scale with the same cuts.

Table 2. Total number of cluster bright objects $\left(N_{\text {brightobj }}\right)$ of detected sources in $H 160$ images $\left(N_{\text {Hdetect }}\right)$ and of new sources in IR stack images $\left(N_{\text {IRdetect }}\right)$.

\begin{tabular}{lclc}
\hline \hline Image & $N_{\text {bright }}$ & $N_{\text {Hdetect }}$ & $N_{\text {IRdetect }}$ \\
\hline M0717cl & 14 & 3096 & 972 \\
M0717par & 0 & 2181 & 1266 \\
M01149cl & 23 & 3379 & 972 \\
M01149par & 0 & 2270 & 1133 \\
\hline
\end{tabular}

those at redshift $6-8$. Table 2 lists the total number of sources detected after each step. In the final catalogues these IR-detected objects are identified as ID $=20000+$ their original ID.

The combined detection catalogue was then used to obtain the photometric measurement (both aperture and total photometry) in the other HST bands using SExtractor on processed images convolved to $H 160$ resolution $\left(0.18^{\prime \prime}\right)$ with a convolution kernel obtained taking the ratio of the PSFs of the two images in the Fourier space.

We assessed the detection completeness as a function of the $H$-band magnitude by running simulations with synthetic sources. We first generated populations of point-like and exponential profile sources with total $H$-band magnitude in the range 26.5-30.0 mag. Disk-like sources were assigned an input half-light radius, $R_{\mathrm{h}}$, randomly drawn from a uniform distribution between 0.0 and 1.0 arcsec. At each run, we placed 200 of these fake galaxies at random positions in our detection image, avoiding positions where real sources are observed on the basis of the original SExtractor segmentation map. We then performed the detection on the simulated image, using the same SExtractor parameters adopted in the real case. Figure 2 shows the completeness as a function of the total input magnitude of various simulated objects (both point- and disk-like). We find that the $90 \%$ detection completeness for the point sources is at $H \sim$ 27.2(27.8) for M0717(M1149) and decreses to $H \sim 26.5(26.6)$ and $H \sim 25.7(26.3)$ for disk-like galaxies of $R_{\mathrm{h}}=0.2$ arcsec and $R_{\mathrm{h}}=0.3$ arcsec, respectively.

\section{3. $\mathrm{K}$ and IRAC photometry with T-PHOT}

We obtained $\mathrm{K}$ and IRAC photometry via a template-fitting technique with T-PHOT (Merlin et al. 2015, 2016b) using galaxy shapes in the detection band $H 160$ as priors. Please check for these throughout and correct accordingly. For this purpose we took advantage of T-PHOT V2.0, which allows us to simultaneuosly use as templates the observed galaxy shapes (for all faint objects) and the analytic profiles for the ICL and bright cluster galaxies. In the latter case, after some tests, we decided to fix 
M. Di Criscienzo et al.: The ASTRODEEP Frontier Fields catalogues. III.
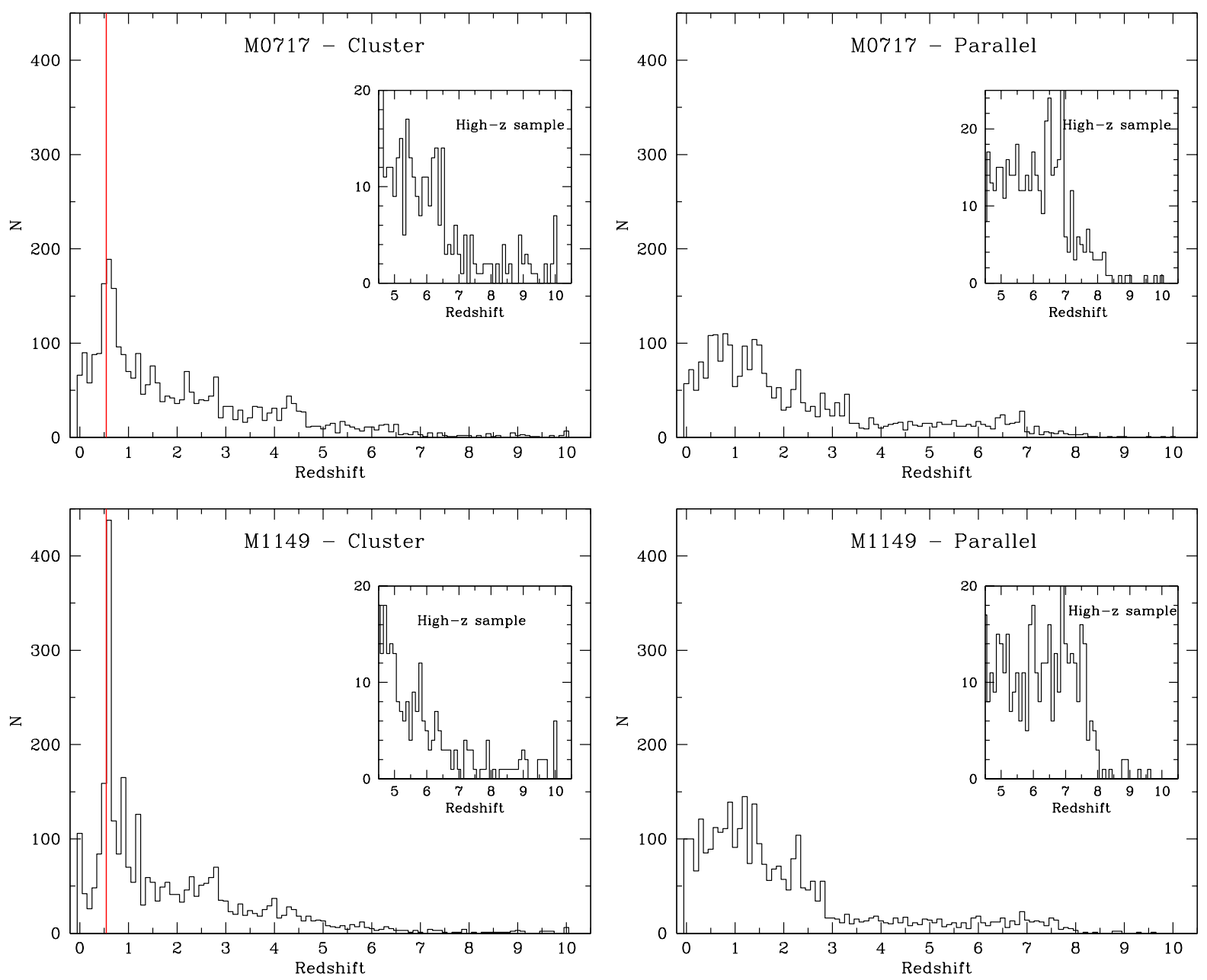

Fig. 4. Photometric redshift distribution in our four catalogues. The vertical red line indicates the redshift of the clusters. Insets show a zoom for object with $z>5$ to appreciate the high redshift tail of the distribution.

the ratio between the two components (when present) used for the analytic fits to avoid possible degeneracy issues in the fitting procedure.

As discussed at length in Merlin et al. (2015), the segmentation of the objects obtained by SExtractor map may be too small to capture the whole galaxy shape, potentially leading to biases in the flux estimate with T-PHOT. In order to minimize this effect, we dilated the SExtractor output map with the same procedure described in Galametz et al. (2013) before feeding this map to T-PHOT, thereby enlarging the size of the segmented area of each source by a given factor, depending on the original area. We then prepared the measurement image by applying, to the rms and background, a corrective factor via injection of fake PSF-shaped sources in about 200 positions in empty regions without detected sources. After measuring the flux of the fake point sources injected at the selected positions, we computed the rms map multiplicative factor required to make the distribution of the measured $\mathrm{S} / \mathrm{N}$ having standard deviation consistent with 1 . Instead of deriving the correcting factor for the background, we measured the shift of the mean of the distribution of the fake sources on copies of the images with small constant artificial background offsets and computed the offset required to make the measured shift consistent with zero. In the case of the $K$-band images, to take into account the noise correlation we added a further correcting factor for the background to be consistent with the magnitudes published in Brammer et al. (2016).
Following the procedure used to derive the photometric catalogue of MACS-J0416 and Abell-2744, we also estimated a local background for each source and combined all the measurements to build a global background image, which was then subtracted from the original image. Figure 3 shows the residual images obtained by subtracting the scaled models generated by T-PHOT compared with original $K$ and IRAC images of M0717.

We follow the same strategy to process the parallel fields; needless to say there is no need to include any analytical model in the priors list.

\section{Results}

We distribute final complete multiwavelength photometric catalogues of four fields (two centred on clusters M07171 and M1149 + two parallel fields), which contains 10 bands fluxes and magnitudes, and corresponding uncertainties. All the fluxes were finally corrected for galactic exinction derived with Schlegel et al. (1998) dust emission maps. A flag (called RELFLAG) is associated with each object, which gives an indication of the robustness of photometric estimates. "Good sources" have RELFLAG $=1$, which means they have more than 5 HST bands with reliable (Sextractor's internal FLAGS $\leq 16$ ) flux measurement available. As in Castellano et al. (2016a) we complement the publicly released catalogues with photometric redshift, stellar mass, and star formation rate as described below. 

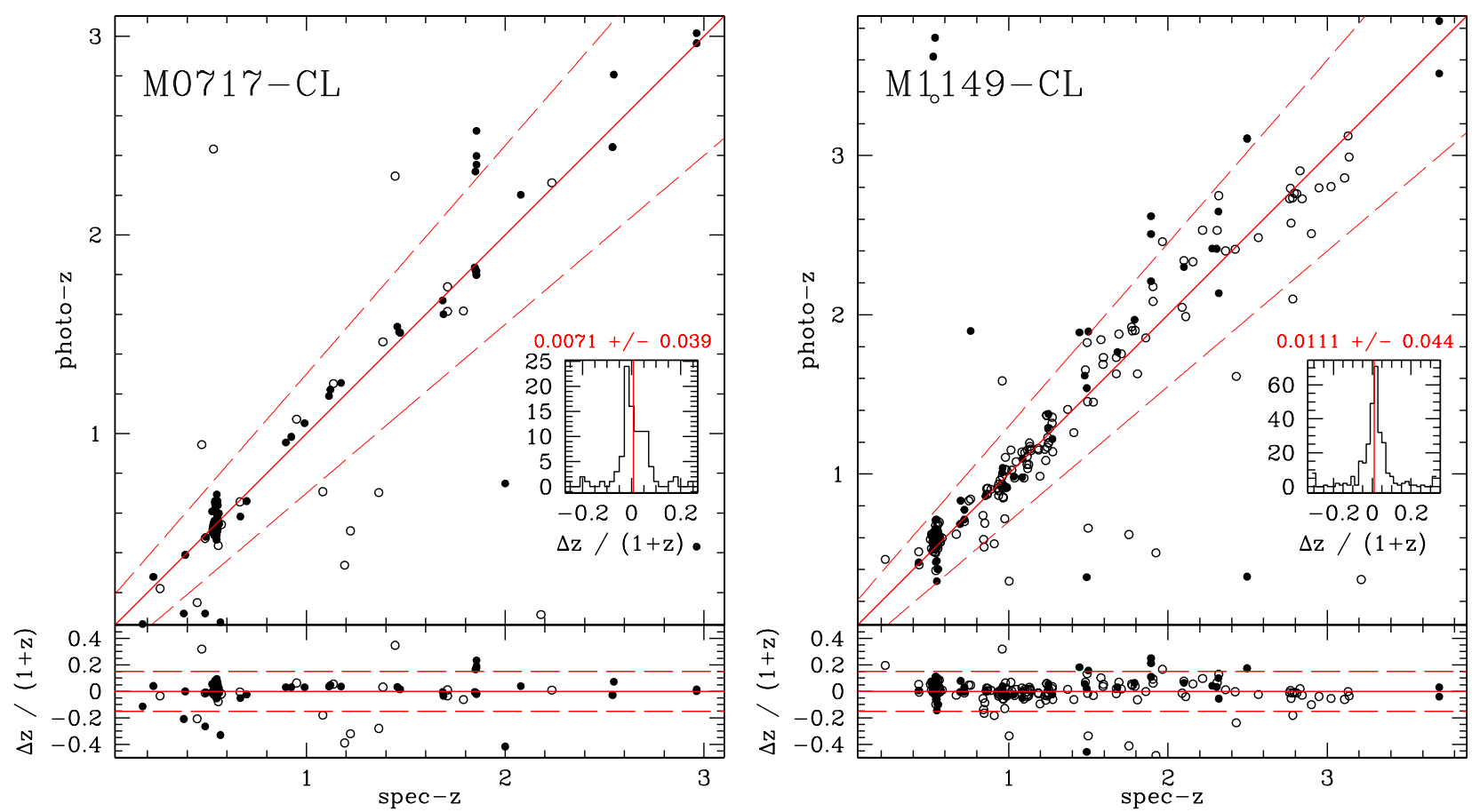

Fig. 5. Comparison between photometric median redshifts of our good sources (RELFLAG $=1)$ and the spectroscopic estimate for M0717 $(l e f t)$ and M1149 (right). Filled circles represent best quality spectroscopic redshifts $(Q=4)$. In the lower panels we show $\Delta z /(1+z)=\left(z_{\text {spec }}-z_{\text {phot }}\right) /\left(1+z_{\text {spec }}\right)$ as a function of the spectroscopic redshift. In the inner small panels the distribution of $\Delta z /(1+z)$ is shown together with its average (vertical line) and rms after excluding outliers, as discussed in the text.

\subsection{Photometric redshifts and comparison with spectroscopic samples}

To minimize systematic effects due to the use of a single method we measured photometric redshifts using six different algorithms: 1) OAR (Castellano et al. 2016a); 2) McLure (McLure et al. 2011); 3) Mortlock (Arnouts et al. 1999; Blanton \& Roweis 2007); 4) Parsa (Arnouts et al. 1999; Ilbert et al. 2006); 5) Marmol-Queralto-1 (Brammer et al. 2008; Blanton \& Roweis 2007); and 6) Marmol-Queralto-2 (Brammer et al. 2008; Fioc \& Rocca-Volmerange 1997). These techniques are described in detail in Sect. 3 of Castellano et al. (2016a). Photometric redshifts are determined for all good sources using all available bands with the exception of $K$ and IRAC fluxes, which are unreliable due to severe blending with other sources (T-PHOT parameter MaxCvRatio > 1.0; see Merlin et al. 2016). In Fig. 4 we show the resulting median photometric redshifts distribution computed for all "good sources".

Objects that have a positive match (within 1 arcsec) with reliable public spectroscopic samples are assigned the measured spectroscopic redshift. In particular, we consider the redshifts from the public dataset by Ebeling et al. (2014), GLASS (Treu et al. 2015, for sources with quality flag $Q=3$ and $Q=4$ ) together with the arcs from Limousin et al. (2012) in the case of M0717 and Smith et al. (2009) for M1149. In GLASS catalogues the number of objects with $z$ determination (and $Q=3,4$ ) in MACS1149 is about three time larger than in MACS0717 and these differences are maintained when a cross-correlation with our sample is made (see Fig. 5).

When compared with spectroscopic results, median values of photometric redshift are more accurate than the individual runs computed with the six different techniques $(0.046 \leq \mathrm{rms} \leq$ 0.055 ), and for this reason we give the median value in the released catalogue. In Fig. 5 we show the comparison between our median estimate of photometric redshift and spectroscopic

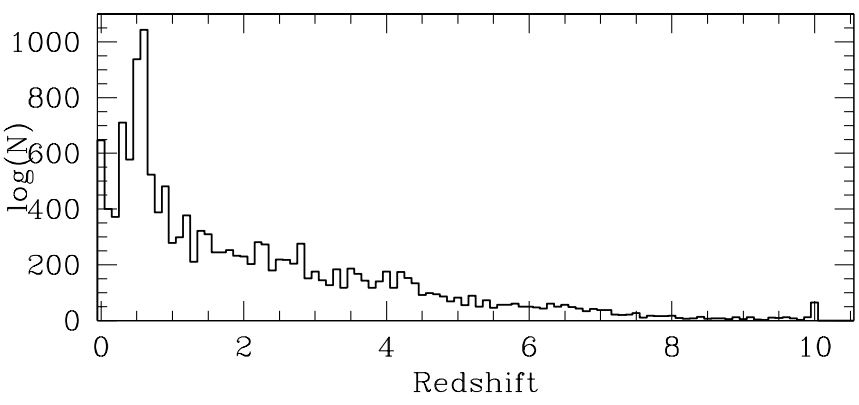

Fig. 6. Distribution of photometric redshift of all good sources detected in the four clusters.

Table 3. Photomeric redshift accuracy.

\begin{tabular}{ccccc}
\hline \hline Field & Spec. sample & Outliers & $\langle\Delta z /(1+z)\rangle$ & $\sigma_{\Delta z /(1+z)}$ \\
\hline M0717 & 109 & $18 \%$ & 0.0071 & 0.037 \\
M1149 & 285 & $9 \%$ & 0.011 & 0.044 \\
\hline
\end{tabular}

value for all our good sources in the cluster's field. Following Dahlen et al. (2013) we define as outliers all sources having $|\Delta z| /(1+z)=\left|\left(z_{\text {spec }}-z_{\text {phot }}\right)\right| /\left(1+z_{\text {spec }}\right) \geq 0.15$. In Table 3 we report the number of outliers and the statistic in each cluster.

In the case of the parallel fields the final sample includes only two objects with spectroscopic redshift and it makes no sense to provide the statistics.

In Fig. 6 we show the distribution of photometric redshift for all objects of the first four FFs (Castellano et al. 2016; + this work). 
M. Di Criscienzo et al.: The ASTRODEEP Frontier Fields catalogues. III.
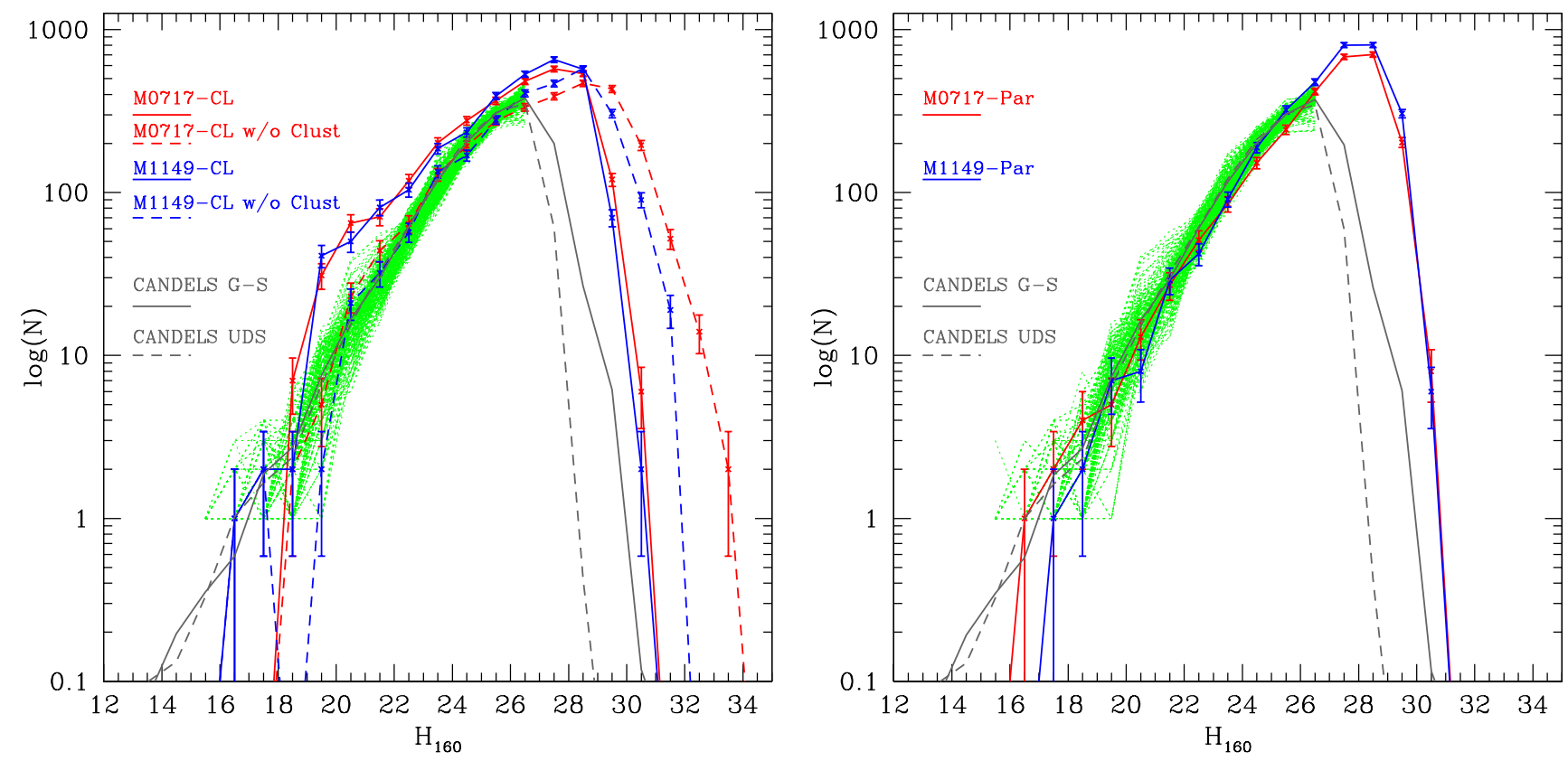

Fig. 7. Demagnified number counts in the cluster fields when sources with $z_{\text {phot }}$ within 0.1 the redshift of the relative cluster are removed (solid lines). As a comparison, number counts normalized to the same FF area from the public CANDELS GOODS-South and UDS catalogues are shown. The green lines in particular are number counts from randomly chosen portions having the same area of the FF pointings.

\subsection{Demagnified number counts and rest-frame physical properties}

Ultra-deep IR observations of the FF in combination with the strong gravitational lensing effect allow us to probe stellar masses and star formation rates at unprecedented low limits. We first determined magnification values from all available lensing models described in detail on the FF website ${ }^{5}$ on an object-byobject basis taking into account source position and redshifts. We assigned a magnification to each source in our catalogues as the median values computed with the available lensing models. The magnified number counts are shown in Fig. 7 compared with total number counts from CANDELS GOODS-South (Guo et al. 2013) and UDS (Galametz et al. 2013) surveys normalized to FF area. For magnitudes brighter then $H 160=26$ mag, the number counts are consistent with the CANDELS counts once magnification is taken into account and when sources with $z_{\text {phot }}$ within 0.1 the redshift of the relative cluster are removed. At fainter magnitudes the FF cluster pointings allow us to detect sources up to 4 mag intrinsically fainter than objects in the deepest areas of the CANDELS fields. Figure 8 shows the comparison with Abel-2744 and MACS-J0416.

Finally we also release de-magnified $M_{\text {star }}$ and SFRs as a function of redshift for galaxies in our catalogues obtained through SED fitting. Galaxy properties are computed by fitting Bruzual \& Charlot (2003) templates with our custom zphot. exe code (Giallongo et al. 1998; Fontana et al. 2000; Grazian et al. 2006) at the previously determined median photometric redshift. In the $\mathrm{BCO} 3$ fit we assume exponentially declining star formation histories with e-folding time $0.1 \leq$ $\tau \leq 15$, a Salpeter (1955) initial mass function, and we allow both Calzetti et al. (2000) and Small Magellanic Cloud (Prevot et al. 1984) extinction laws. We fit all the sources both with stellar emission templates only and including the contribution from nebular continuum and line emission following

\footnotetext{
5 http://www.stsci.edu/hst/campaigns/frontier-fields/ Lensing-Models
}

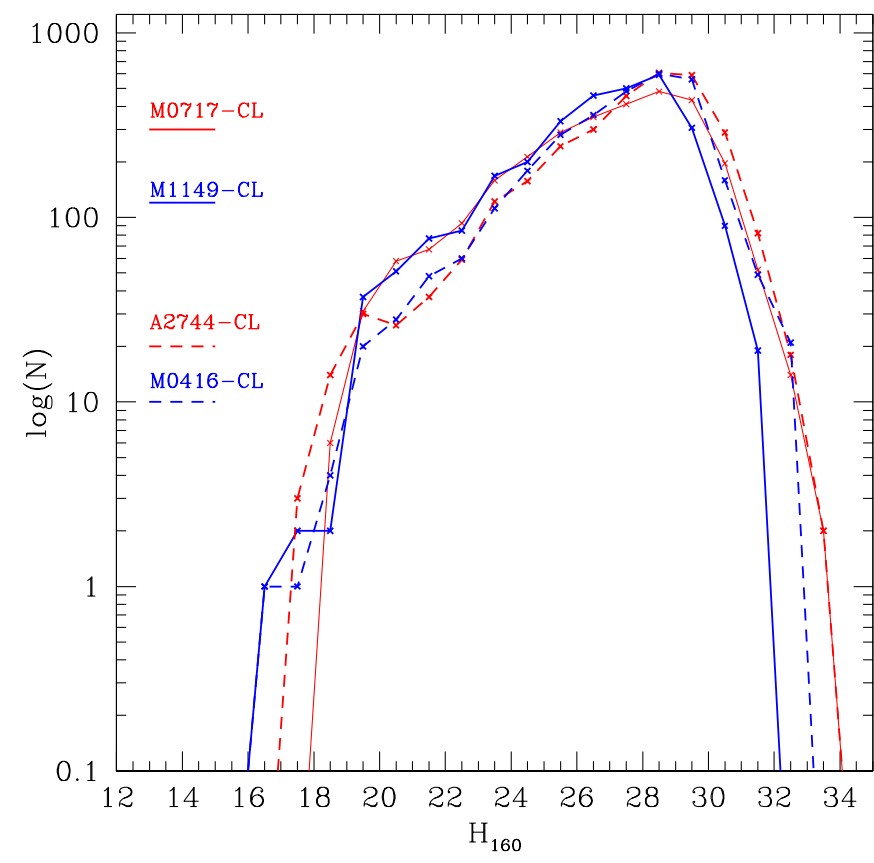

Fig. 8. Demagnified number counts in the cluster fields investigated in this work compared with the previous two FFs from Castellano et al. (2016).

Schaerer \& de Barros (2009) under the assumption of an escape fraction of ionizing photons fesc $=0.0$ (see also Castellano et al. 2014). The FFs allow us to probe the galaxy distribution down to very low masses and SFRs, including objects with $M_{\star} \sim 10^{7} M_{\odot}$ and $S F R \sim 0.1-1 M_{\odot} \mathrm{yr}^{-1}$ at $z>6$, depending on magnification.

\section{Conclusions}

We presented the public release of multiwavelength photometry of the Frontier Fields M0717 and M1149 (cluster and parallel 
pointings) including optical and NIR ACS and WFC3, MOSFIRE Ks, and IRAC 3.6 and 4.5 IRAC bands. We followed the same method used and described in detail in Merlin et al. (2016) for Abell-2744 and MACS-J0416 with small differences mainly due to the extreme crowding of the two investigated clusters. The catalogues also report first high-level data products such as photometric redshifts, magnification factors, and rest-frame properties for the detected objects, which can be downloaded from the ASTRODEEP website ${ }^{6}$. This work, as presented in the first two papers (see e.g. Vanzella et al. 2017a,b), aims to provide a reference for future investigations of extragalactic populations.

Acknowledgements. The authors acknowledge the contribution of the FP7 SPACE project ASTRODEEP (Ref. No. 212725) supported by European Commission. R.A. acknowledges the support from the ERC Advanced Grant "QUENCH".

\section{References}

Arnouts, S., Cristiani, S., Moscardini, L., et al. 1999, MNRAS, 310, 540

Barden, M., Häußler, B., Peng, C. Y., McIntosh, D. H., \& Guo, Y. 2012, MNRAS, 422, 449

Bertin, E., \& Arnouts, S. 1996, A\&AS, 117, 393

Binney, \& Tremaine 1987, Nature, 326, 219

Blanton, M. R., \& Roweis, S. 2007, AJ, 133, 734

Bouwens, R. J., Oesch, P. A., Labbé, I., et al. 2016, ApJ, 830, 67

Bouwens, R. J., Illingworth, G. D., Oesch, P. A., et al. 2017, ApJ, 843, 41

Brammer, G. B., van Dokkum, P. G., \& Coppi, P. 2008, ApJ, 686, 1503

Brammer, G. B., Marchesini, D., Labbé, I., et al. 2016, ApJS, 226, 6

Calzetti, D., Armus, L., Bohlin, R. C., et al. 2000, ApJ, 533, 682
Castellano, M., Sommariva, V., Fontana, A., et al. 2014, A\&A, 566, A19 Castellano, M., Amorín, R., Merlin, E., et al. 2016a, A\&A, 590, A31 Castellano, M., Yue, B., Ferrara, A., et al. 2016b, ApJ, 823, L40 Dahlen, T., Mobasher, B., Faber, S. M., et al. 2013, ApJ, 775, 93 Fontana, A., D'Odorico, S., Poli, F., et al. 2000, AJ, 120, 2206 Fioc, M., \& Rocca-Volmerange, B. 1997, A\&A, 326, 950 Ebeling, H., Ma, C.-J., \& Barrett, E. 2014, ApJS, 211, 21

Galametz, A., Grazian, A., Fontana, A., et al. 2013, ApJS, 206, 10 Giallongo, E., D’Odorico, S., Fontana, A., et al. 1998, AJ, 115, 2169 Grazian, A., Fontana, A., de Santis, C., et al. 2006, A\&A, 449, 95 Guo, Y., Ferguson, H. C., Giavalisco, M., et al. 2013, ApJS, 207, 24 Ilbert, O., Arnouts, S., McCracken, H. J., et al. 2006, A\&A, 457, 841 Limousin, M., Ebeling, H., Richard, J., et al. 2012, A\&A, 544, A71 Livermore, R. C., Finkelstein, S. L., \& Lotz, J. M. 2017, ApJ, 835, 113 Lotz, J. M., Koekemoer, A., Coe, D., et al. 2017, ApJ, 837, 97

McLeod, D. J., McLure, R. J., \& Dunlop, J. S. 2016, MNRAS, 459, 3812 McLure, R. J., Dunlop, J. S., de Ravel, L., et al. 2011, MNRAS, 418, 2074 Menci, N., Merle, A., Totzauer, M., et al. 2017, ApJ, 836, 61

Merlin, E., Fontana, A., Ferguson, H. C., et al. 2015, A\&A, 582, A15

Merlin, E., Amorín, R., Castellano, M., et al. 2016a, A\&A, 590, A30 Merlin, E., Bourne, N., Castellano, M., et al. 2016b, A\&A, 595, A97

Peng, C. Y., Ho, L. C., Impey, C. D., \& Rix, H.-W. 2011, Astrophysics Source Code Library [record ascl: 1104.010 ]

Prevot, M. L., Lequeux, J., Prevot, L., Maurice, E., \& Rocca-Volmerange, B. 1984, A\&A, 132, 389

Santini, P., Ferguson, H. C., Fontana, A., et al. 2015, ApJ, 801, 97

Schaerer, D., \& de Barros, S. 2009, A\&A, 502, 423

Schlegel, D. J., Finkbeiner, D. P., \& Davis, M. 1998, ApJ, 500, 525

Smith, G. P., Ebeling, H., Limousin, M., et al. 2009, ApJ, 707, L163

Treu, T., Schmidt, K. B., Brammer, G. B., et al. 2015, ApJ, 812, 114

Vanzella, E., Calura, F., Meneghetti, M., et al. 2017a, MNRAS, 467, 4304

Vanzella, E., Castellano, M., Meneghetti, M., et al. 2017b, ApJ, 842, 47

Zheng, W., Zitrin, A., Infante, L., et al. 2017, ApJ, 836, 210

\footnotetext{
6 At http://www.astrodeep.eu/ff34 or at http://astrodeep. $\mathrm{u}$-strasbg.fr//ff//index.html
} 